\title{
QUANTUM LOGICS WITH LATTICE STATE SPACES
}

\author{
JIŘ́́ BINDER AND MIRKO NAVARA
}

\begin{abstract}
Let $L$ be a quantum logic and let $S(L)$ denote the set of all states on $L$. (By a state we mean a nonnegative bounded $\sigma$-additive measure, not necessarily normalized.) We ask whether every logic whose state space is a lattice has to be Boolean. We prove that this is so for finite logics and "projection logics." On the other hand, we show that there exist even concrete non-Boolean logics with a lattice state space (in fact, we prove that every countable concrete logic can be enlarged to a logic with a lattice state space). In the appendix we shortly consider the lattice properties of the set of observables and correct the paper [10].
\end{abstract}

1. Introduction and preliminaries. In axiomatic quantum mechanics (see $[3,8])$, the "event structure" of a physical system is modelled by a $\sigma$-orthomodular partially ordered set (usually called a quantum logic). The states of the system then may be assumed to correspond to nonnegative bounded $\sigma$-additive measures on $L$, called "states" on $L$. Let us denote by $S(L)$ the set of all states on $L$. If $L$ is a Boolean $\sigma$-algebra, which is the case of an experiment subject to "classical mechanics," then $S(L)$ is a lattice. In this paper we speculate whether this property of $S(L)$ characterizes exactly "classical experiments." We find an axiomatic setup of the problem and obtain the results indicated in the abstract.

Let us first review basic notions as we shall use them in the sequel.

DEFINITION 1. A (quantum) logic is a set $L$ equipped with a partial ordering $\leq$ and a unary operation ', such that the following conditions are satisfied (the symbols $\wedge, \vee$ stand for the lattice operations induced by $\leq$ ):

(1) $0,1 \in L$,

(2) $a \leq b \Rightarrow b^{\prime} \leq a^{\prime}$ for any $a, b \in L$,

(3) $\left(a^{\prime}\right)^{\prime}=a$ for any $a \in L$,

(4) if $a_{n}(n \in N)$ is a countable subset of $L$ such that $a_{n} \leq a_{m}^{\prime}$ whenever $n \neq m$, then $\bigvee_{n \in N} a_{n}$ exists in $L$, and

(5) if $a, b \in L$ and $a \leq b$ then $b=a \vee\left(b \wedge a^{\prime}\right)$.

Obviously, a logic need not be distributive and need not be a lattice. Most frequently used examples of logics are Boolean $\sigma$-algebras and lattices of projections in a von Neumann algebra.

DEFINITION 2. A state on a logic $L$ is a nonnegative bounded mapping $s: L \rightarrow R$ such that

(1) $s(0)=0$, and

(2) whenever $a_{n}(n \in N)$ is a sequence in $L$ such that $a_{n} \leq a_{m}^{\prime}$ for $n \neq m$, then $s\left(\bigvee_{n \in N} a_{n}\right)=\sum_{n \in N} s\left(a_{n}\right)$.

Received by the editors June 26, 1986.

1980 Mathematics Subject Classification (1985 Revision). Primary 81B10, 06C15; Secondary 46E27, 81C20. 
Let us denote by $S(L)$ the set of all states on $L$. If we order $S(L)$ "pointwise" (and assume that $S(L) \neq \varnothing$, of course), we obtain a partially ordered set with a least element. We shall always view $S(L)$ this way. Observe that $S(L)$ is closed under the formation of sums and nonnegative multiples.

In what follows, we shall be mainly concerned with the lattice properties of $S(L)$. Notice that $S(L)$ is a lattice once each pair $s, t \in S(L)$ has the least upper bound in $S(L)$. Indeed, a simple verification gives that if $s \vee t$ is the least upper bound for $s, t$ in $S(L)$, then $s+t-(s \vee t)$ is the greatest lower bound for $s, t$ in $S(L)$.

2. Results. The departure point of our consideration is the following theorem.

THEOREM 1. If $L$ is a Boolean $\sigma$-algebra, then $S(L)$ is a lattice.

ProOF. Suppose that we are given two states $s, t \in S(L)$. We claim that the mapping $r: L \rightarrow R$, defined by the formula $r(a)=\sup _{b \in L}\left[s(b \wedge a)+t\left(b^{\prime} \wedge a\right)\right]$, is the least upper bound for $s, t$ in $S(L)$. To show this, let us first verify that $r$ is a state. Clearly $r(0)=0$, and a routine calculation gives that $r$ is additive. Suppose now that $a_{n}(n \in N)$ is a sequence of mutually orthogonal elements in $L$. Suppose that we are given an $\varepsilon>0$. Then there exists $k \in N$ such that $\sum_{n \geq k} s\left(a_{n}\right)<\varepsilon$ and $\sum_{n \geq k} t\left(a_{n}\right)<\varepsilon$. Making use of the additivity for $r$, we obtain

$$
\begin{aligned}
r\left(\bigvee_{n \in N} a_{n}\right) & =\sup _{b \in L}\left[s\left(b \wedge \bigvee_{n \in N} a_{n}\right)+t\left(b^{\prime} \wedge \bigvee_{n \in N} a_{n}\right)\right] \\
& =\sup _{b \in L} \sum_{n \in N}\left[s\left(b \wedge a_{n}\right)+t\left(b^{\prime} \wedge a_{n}\right)\right] \leq \sum_{n \in N} r\left(a_{n}\right) \\
& \leq \sum_{n \leq k-1} r\left(a_{n}\right)+\sum_{n \geq k}\left[s\left(a_{n}\right)+t\left(a_{n}\right)\right]<r\left(\bigvee_{n \in N} a_{n}\right)+2 \varepsilon
\end{aligned}
$$

Since the latter inequality is fulfilled for an arbitrary $\varepsilon>0$, we have shown that $r$ is $\sigma$-additive. Therefore $r \in S(L)$ and the proof is complete.

A natural question is whether a logic with a lattice state space must be Boolean. We first examine finite logics. Obviously, the problem degenerates if $S(L)$ is very "poor" (which, unfortunately, may actually happen; see $[2,5]$ ). Following the usual terminology (see [3]), let us call a logic $L$ quite full if for any $a, b \in L$ such that $b \not a$ there exists a state $s \in S(L)$ with $s(a)=0$ and $s(b)=1$. We now have the following result.

THEOREM 2. Let $L$ be a finite quite full logic. Then $S(L)$ is a lattice if and only if $L$ is a Boolean algebra.

Proof. Suppose that $L$ is a finite quite full logic and suppose that $S(L)$ is a lattice. We are going to show that $L$ is Boolean. Since $L$ is finite, it suffices to prove the following property of $L$ : If $a, b$ are atoms in $L$ then either $a=b$ or $a \leq b^{\prime}$. Suppose to the contrary that there are atoms $a, b \in L$ fulfilling neither of the latter two conditions. Since $L$ is quite full, there exist states $s, t \in S(L)$ such that $s(b)=t\left(b^{\prime}\right)=0, s(a)=t(a)=1$. Suppose that $u$ is the least upper bound for $s$ and $t$ in $S(L)$. The state $s+t$ is an upper bound for $s$ and $t$ and therefore $u(x) \leq s(x)+t(x)$ for all $x \in L$. For any $c \in L \backslash\{0, a\}$ we have $c \not a$ and hence we can find a state $s_{c} \in S(L)$ such that $s_{c}(a)=0$ and $s_{c}(c) \geq t(c)$. Put 
$v=s+\sum_{c \in L \backslash\{0, a\}} s_{c}$. Then $v$ is an upper bound for $s$ and $t$. Moreover, we have $s \leq u \leq v$ and $v(a)=s(a)$. This implies $u(a)=s(a)$. Finally, we obtain

$$
\begin{aligned}
u(1) & =u(a)+u\left(a^{\prime}\right)=s(a)+u\left(a^{\prime}\right)<s(a)+t(a)+u\left(a^{\prime}\right) \\
& \leq s(a)+s\left(a^{\prime}\right)+t(a)+t\left(a^{\prime}\right)=s(1)+t(1)=s\left(b^{\prime}\right)+t(b) \\
& \leq u\left(b^{\prime}\right)+u(b)=u(1) .
\end{aligned}
$$

This is a contradiction. Therefore every two distinct atoms $a, b \in L$ satisfy $a \leq b^{\prime}$ and the proof is complete.

Let us now examine the logic $P(A)$ of all projections in a von Neumann algebra $A$.

THEOREM 3. Let $A$ be a von Neumann algebra without a type $\mathrm{I}_{2}$ direct summand acting on a separable Hilbert space. Let $L=P(A)$ be the logic of all projections in A. Then $P(A)$ is a Boolean $\sigma$-algebra if and only if $S(L)$ is a lattice.

Proof. We have to show that if $S(L)$ is a lattice, then $A$ is commutative. Using the results of $[\mathbf{1}, \mathbf{9}], s$ is a state on the logic $P(A)$ if and only if $s$ is a restriction of a (unique) normal positive functional on $A$. Since each normal hermitian functional $f$ on $A$ possesses a Jordan decomposition $f=f^{+}-f^{-}$into positive parts, it follows that there is a one-to-one correspondence between the set $S(L)-S(L)$ of all signed measures on $L$ and the set $N_{h}$ of all normal hermitian functionals on $A$. Clearly, this correspondence preserves the linear and order structure. If $S(L)$ is a lattice then so is $N_{h}$. Since the Jordan decomposition fulfils the equality $\|f\|=\left\|f^{+}\right\|+\left\|f^{-}\right\|$, the set $N_{h}$ is a normed lattice. Consequently, the dual of $N_{h}$ (which is the real linear space $A_{h}$ of all hermitian operators in $A$ ) is a lattice. This property is satisfied only when $A$ is commutative [4]. The proof is complete.

A question now arises whether each quite full logic with a lattice state space must be Boolean. We shall answer this question in the negative. We shall show in fact that counterexamples can be constructed even in the class of set-representable logics. We call these logics concrete (see also $[3,6]$ ). Let us recall that a concrete logic is a pair $L=(X, C)$, where $X$ is a nonempty set and $C$ is a collection of subsets of $X$ subject to the following conditions:

(1) $\varnothing \in C$,

(2) if $M \in C$ then $X \backslash M \in C$, and

(3) if $M_{n}(n \in N)$ is a mutually disjoint sequence in $C$ then $\bigcup_{n \in N} M_{n} \in \mathcal{C}$.

Obviously, each concrete logic is a (quite full) logic. A concrete logic may have many intrinsically equivalent representations. We say that a (concrete) logic $(Y, D)$ is a representation of a logic $(X, C)$ if there is a one-to-one mapping $f: C \rightarrow D$ such that

(a) $f(\varnothing)=\varnothing$,

(b) $f(X \backslash A)=Y \backslash f(A)$ for each $A \in C$, and in $C$.

(c) $f\left(\bigcup_{n \in N} A_{n}\right)=\bigcup_{n \in N} f\left(A_{n}\right)$ for each mutually disjoint sequence $A_{n}(n \in N)$

Obviously, if $(Y, D)$ is a representation of $(X, C)$ then $S(D), S(C)$ are equivalent as partially ordered sets.

We are going to show that there are concrete logics with a lattice state space and which are in a sense arbitrarily far from Boolean algebras. We shall need the 
following auxiliary notion. Let $(X, C),(X, D)$ be concrete logics. We say that $(X, D)$ is an enlargement of $(X, C)$ if the following two conditions hold:

(i) $C \subset D$,

(ii) if $A, B \in C$ then the set $A \cap B$ belongs to both $C, D$ or to neither of them.

Our final result says that in many cases a concrete logic can be enlarged to a concrete logic with a lattice state space. Since every enlargement of a non-Boolean logic cannot be Boolean (because, of course, a concrete logic $(X, D)$ is Boolean if and only if $A \cap B \in D$ for each couple $A, B \in D$ ), we can thus generate various examples of non-Boolean logics with lattice state spaces. We shall need the following construction:

THEOREM 4. Suppose that $(X, C)$ is a concrete logic with the domain set $X$ countable. Then $(X, C)$ possesses a representation $(Y, \mathcal{E})$ with the following property: We can find an enlargement $(Y, D)$ of $(Y, \mathcal{E})$ and two $\sigma$-algebras $(Y, A),(Y, B)$ of subsets of $Y$ such that $A \subset D \subset B$ and such that each state on $(Y, A)$ has a unique extension to a state on $(Y, B)$.

ProOF. Let $U$ be a set of the first uncountable cardinality. Due to Solovay [7], each finite $\sigma$-additive measure on the $\sigma$-algebra $\exp U$ of all subsets of $U$ is carried by a countable set. Put $Y=U \times X$ and let $(Y, \mathcal{E})$ be the representation of $(X, C)$ given by the mapping $A \rightarrow U \times A$. Let $D$ consist of all sets $M \subset Y$ fulfilling the following property: The set $T(M)$ of all $u \in U$ such that the set $\{x \in X:(u, x) \in M\}$ does not belong to $C$ is at most countable.

First, we shall show that $(Y, D)$ is a concrete logic. Obviously, $\varnothing \in D$. Since $T(Y \backslash M)=T(M)$, the relation $M \in D$ implies $Y \backslash M \in D$. Suppose that $M_{n}(n \in$ $N)$ is a sequence of mutually disjoint sets in $D$. Then $T\left(\bigcup_{n \in N} M_{n}\right) \subset \bigcup_{n \in N} T\left(M_{n}\right)$ and hence $\bigcup_{n \in N} M_{n} \in D$. We have verified that $(Y, D)$ is a set logic. It is routine to check that $(Y, D)$ is an enlargement of $(Y, \mathcal{E})$.

To establish the statement of Theorem 4 , put now $B=\exp Y$ and define $A$ to be the set of all subsets $M$ of $Y$ fulfilling the following property: There are at most countably many $u \in U$ for which the set $\{x \in X:(u, x) \in M\}$ is neither $\varnothing$ nor $X$. Then $A \subset D$ and $A$ is clearly a $\sigma$-algebra.

Let $s \in S(A)$. As $A$ contains the $\sigma$-algebra $\{P \times X: P \subset U\}$, there is a countable set $P_{0} \subset U$ such that $s\left(Y-P_{0} \times X\right)=0$. Since all subsets of $P_{0} \times X$ belong to $A$, we infer that there is a countable set $Q_{0} \subset P_{0} \times X$ such that $s\left(Y-Q_{0}\right)=0$. The state $s$ can be uniquely expressed as a countable convex combination of states on $A$ such that each of them is concentrated in a point of $Q_{0}$. These states have unique extensions to concentrated states on $(Y, B)$ and thus $s$ extends uniquely to the same countable convex combination of the extended states. The proof is complete.

THEOREM 5. Every concrete logic $(X, C)$ with countable domain $X$ has such a representation that admits an enlargement $(Y, D)$ with a lattice state space $S(D)$.

PROOF. It suffices to prove that the state space of the concrete logic $(Y, D)$ obtained in Theorem 4 is a lattice. Each state $s \in S(D)$ extends uniquely to a state $h(s) \in S(B)$. The mapping $h$ is one-to-one and it remains to show that $h$ preserves the ordering of states. If $s, t \in S(D)$ with $s \leq t$, then $t-s$ is a state on $D$ which extends to $h(t-s) \in S(B)$. The state $h(t-s)+h(s)$ is an extension of $t$, hence $h(t)-h(s)=h(t-s)$ and $h(s) \leq h(t)$. The reverse implication is obvious. So $s \leq t$ 
if and only if $h(s) \leq h(t)$. According to Theorem $1, S(B)$ is a lattice and therefore so also is $S(D)$. This completes the proof.

3. Appendix: The lattice structure of the set of observables on a logic. In [10] one introduces the order structure into the set of observables and asks a question formally closed to our interest in this note: Supposing the set of all observables on $L$ is a lattice, does $L$ have to be Boolean? The author answers the question in the positive. However, there is something overlooked in the proof (see p. 349 of $[\mathbf{1 0}]$ - from $w+L_{a b} \leq L_{a}, L_{b}$, it cannot be generally obtained that $\left.w+L_{a b} \leq L_{a b}\right)$. We conclude this note by showing that the above statement is false: There is a (finite concrete lattice) non-Boolean logic whose set of observables is a lattice.

Let us first recall basic notions $[3,10]$. Let $B(R)$ stand for the $\sigma$-algebra of Borel subsets of $R$. By an observable on a logic $L$ we mean a $\sigma$-homomorphism $h: B(R) \rightarrow$ $L$ such that $h(E)=1$ for a bounded set $E \in B(R)$. Each observable $h$ defines a bounded linear functional $\omega$ on $S(L)-S(L)$ by putting $\omega(s)=\int_{-\infty}^{\infty} \lambda d m_{s}(h)(\lambda)$. The set $Y_{0}$ of all such functionals is naturally ordered as a subset of the dual of the linear span of $S(L)$. Obviously, if $L$ is Boolean then $Y_{0}$ is a lattice. The "vice versa" statement is not true as the following simple example shows.

EXAMPLE. Let $L$ be a concrete logic $(X, C)$, where $X=\{1,2,3,4\}$ and $C$ contains $\varnothing,\{1,2\},\{1,3\}$, and their complements. Then the set $Y_{0}$ of all observables on $L$ is a lattice.

PROOF. It is easy to see that for each observable $h: B(R) \rightarrow C$ there is a measurable function $f_{h}: X \rightarrow R$ such that $h(E)=f_{h}^{-1}(E)$ for each $E \in B(R)$. Let $h, k$ be observables and let $f_{h}, f_{k}$ be the functions which represent them. Without any loss of generality we may assume that $f_{h}(1)=f_{h}(2) \leq f_{h}(3)=f_{h}(4)$. If $f_{k}(1)=f_{k}(2)$ (and hence also $\left.f_{k}(3)=f_{k}(4)\right)$ then both $h$ and $k$ are observables on a Boolean subalgebra $\{\varnothing,\{1,2\},\{3,4\}, X\}$ and so $h \vee k$ exists. If $f_{k}(1) \neq f_{k}(2)$ then from the measurability follows that $f_{k}(1)=f_{k}(3), f_{k}(2)=f_{k}(4)$. Changing the role of $f$ and $g$ if necessary and renumbering the elements of $X$ in an appropriate way we may ensure that $f_{k}(3) \leq f_{k}(4)$ and $f_{h}(4) \leq f_{k}(4)$. Then the (measurable) function $f: X \rightarrow R$ defined by putting $f(2)=f(4)=f_{k}(4), f(1)=f(3)=$ $\max \left(f_{h}(3), f_{k}(3)\right)$ is the least upper bound for $f_{h}, f_{k}$ and therefore $f$ represents an observable which becomes the least upper bound for $h, k$. The greatest lower bound can be constructed analogously. This implies that $Y_{0}$ is a lattice.

ACKNOWLEDGMENT. The authors would like to express their gratitude to Professor Pavel Pták for his encouragement during this research.

\section{REFERENCES}

1. E. Christensen, Measures on projections and physical states, Comm. Math. Phys. 86 (1982), 529-538.

2. R. J. Greechie, Orthomodular lattices admitting no states, J. Combin. Theory Ser. A 10 (1971), 119-132.

3. S. Gudder, Stochastic methods in quantum mechanics, North-Holland, New York, 1979.

4. R. V. Kadison, Order properties of bounded self-adjoint operators, Proc. Amer. Math. Soc. 2 (1951), 505-510.

5. P. Pták, Exotic logics, Colloq. Math. (to appear).

6. P. Pták and J. D. M. Wright, On the concreteness of quantum logics, Apl. Mat. 30 (1985), 274-285. 
7. R. M. Solovay, Axiomatic set theory (D. Scott, ed.), Proc. Sympos. Pure Math., vol 13, Part 1, Amer. Math. Soc., Providence, R.I., 1971, pp. 397-428.

8. V. S. Varadarajan, Geometry of quantum theory, vol. I, Van Nostrand, Princeton, N.J., 1968.

9. F. J. Yeadon, Finitely additive measures on projections in finite $W^{*}$-algebras, Bull. London Math. Soc. 16 (1984), 145-150.

10. N. Zierler, Order properties of bounded observables, Proc. Amer. Math. Soc. 14 (1963), 346-351.

Department of Mathematics, Pedagogical Faculty, Charles University, 116 39 Prague 1, Czechoslovakia

Department of Mathematics, Faculty of Electrical Engineering, TeChniCAl University of Prague, 16627 Prague 6, Czechoslovakia 